\title{
Morbilidade em Idosos Dependentes ao Cuidado das Equipas Domiciliárias da Rede Nacional de Cuidados Continuados Integrados na Região de Lisboa e Vale do Tejo: Estudo Transversal Observacional
}

\section{Morbidity in the Dependent Elderly Cared by Home-Teams of the National Network of Continued Integrated Care in the Lisbon and Tagus Valley: Cross-Sectional Study}

\author{
Paula BROEIRO-GONCALVES $\square^{1,2,3}$ \\ Acta Med Port 2017 Jul-Aug;30(7-8):546-554 - https://doi.org/10.20344/amp.8218
}

\section{RESUMO}

Introdução: Em Portugal, a Rede Nacional de Cuidados Continuados Integrados tem como missão dar resposta às novas necessidades de saúde e sociais. Definiu-se como objetivo do estudo conhecer a carga de doença e de dependência dos idosos (75 e mais anos) ao cuidado das equipas de cuidados continuados integrados.

Material e Métodos: Estudo descritivo transversal, numa amostra de 230 participantes, distribuídos por 25 equipas na região de Lisboa e Vale do Tejo, aleatoriamente selecionadas. A colheita de dados decorreu no domicílio dos doentes por entrevista ao cuidador. As variáveis estudadas foram: sociodemográficas; determinantes de incapacidade; grau (escala de Barthel) e duração de dependência; morbilidade (diagnósticos, número e índice de Charlson).

Resultados: A população em estudo tinha: em média, 84 anos; baixa escolaridade (88,7\%); dependência durante 42 meses, grave em $65,2 \%$; em média, 9,5 diagnósticos por pessoa e um índice de Charlson de 8,48 . Os determinantes de incapacidade mais frequentes foram: demência, acidente vascular cerebral e fratura do fémur. Os diagnósticos mais frequentes foram: osteoartrose, hipertensão e demência.

Discussão: Os resultados revelaram uma elevada carga de doença (Charlson de 8,48) e de dependência. Apesar dos diagnósticos serem os esperados e comparáveis com os dados da literatura, a coexistência de dois ou mais foi universal, em média 9,5 por pessoa, afetando diferentes aparelhos/sistemas. A multimorbilidade, a par duma elevada dependência, acarreta desafios clínicos e organizacionais, bem como a necessidade de estudos populacionais.

Conclusão: A população ao cuidado das equipas de cuidados continuados integrados é de risco: idosa, com baixa escolaridade, com elevada carga de doença e de dependência.

Palavras-chave: Assistência de Longa Duração; Avaliação Geriátrica; Comorbidade; Idoso Fragilizado; Idosos, 80 anos ou mais; Instituição de Longa Permanência para Idosos; Portugal

\section{ABSTRACT}

Introduction: In Portugal, the National Network of Continuing Integrated Care's mission is to take care of new health and social needs. The aim of the study was to know the disease burden and disability of the elderly ( 75 and over) cared by the integrated continuing care teams.

Material and Methods: A cross-sectional study carried out in a sample of 230 participants, from 25 teams randomly selected in the region of Lisbon and Tagus Valley. Data were collected at the patient's home trough caregiver's interviewing. The variables studied were: socio-demographic; disability determinants; degree (Barthel's scale) and duration of disability; morbidity (diagnoses, number and Charlson index).

Results: The study population had: on average 84 years; low or no scholar degree level (88.7\%); on average 9.5 problems per person and a Charlson index of 8.48; disability over 42 months (severe in $65.2 \%$ ). The most frequent disability-determinants were: dementia, stroke and femur fracture. The most frequent diagnoses were: osteoarthritis, hypertension and dementia.

Discussion: The results revealed a high disease burden (Charlson of 8.48) and disability. Although the diagnoses were those expected and comparable with the literature, their coexistence was universal, averaging 9.5 per person, affecting different organs/systems. Multimorbidity, coupled with severe disability, leads to clinical and organizational-care challenges, as well as the need for other population base studies.

Conclusion: The population cared by the integrated continuing care teams is at risk: elderly, with low scholar degree level, with a high disease-burden and disability.

Keywords: Aged, 80 and over; Frail Elderly; Homes for the Aged; Long-Term Care; Portugal

\section{INTRODUÇÃO}

A população europeia e mundial está a envelhecer, com mais pessoas - especialmente mulheres - a viverem além dos 80 anos de idade. Desde 2004, na Europa, existem mais pessoas com idade acima de 65 anos do que com menos de 15 anos. ${ }^{1}$ Portugal revela a mesma tendência de envelhecimento demográfico com indicadores que apontam

1. Unidade de Cuidados de Saúde Primários dos Olivais. Agrupamento de Centros de Saúde de Lisboa Central. Lisboa. Portugal.

2. Departamento de Saúde Pública. Escola Nacional de Saúde Pública. Universidade Nova de Lisboa. Lisboa. Portugal.

3. Departamento de Medicina Geral e Familiar. Faculdade de Medicina de Lisboa. Universidade de Lisboa. Lisboa. Portugal.

$\square$ Autor correspondente: Paula Broeiro Gonçalves. paulabroeiro@gmail.com

Recebido: 21 de agosto de 2016 - Aceite: 11 de abril de 2017 | Copyright @ Ordem dos Médicos 2017 
para a melhoria da esperança de vida à nascença e da longevidade..$^{2,3}$

Os adultos de mais idade têm, em geral, vários problemas de saúde crónicos e nenhum deve ser avaliado e tratado isoladamente. A comorbilidade é uma entidade semelhante a multimorbilidade, ambas referindo-se à 'co-ocorrência de duas doenças ou condições médicas numa pessoa' (Tabela 1). Os defensores do conceito de multimorbilidade tendem a concentrar-se na prática dos cuidados de saúde primários, onde a identificação de uma doença índice nem sempre é óbvia nem útil. ${ }^{4,5} \mathrm{O}$ conceito de comorbilidade tem sido usado para transmitir a noção de carga de doença, definida pelo peso total de disfunção fisiológica ou pelo peso total de doenças com impacto sobre um indivíduo. ${ }^{4}$ Atendendo à variabilidade no número e gravidade das comorbilidades entre os idosos, a idade é inadequadamente utilizada como um proxy de carga morbilidade. ${ }^{6} \mathrm{O}$ índice de Charlson é um dos mais amplamente utilizados para medir carga de doença e utiliza morbilidades com diferente impacto na carga total de morbilidade (e.g. ponderação de seis para tumor sólido metastizado, dois para diabetes com complicações), com base no seu risco relativo de morte. ${ }^{7} \mathrm{O}$ índice global ou índice de Charlson permite aferir a comorbilidade (e.g. índice de três ou mais pontos significa alta comorbilidade) e predizer a mortalidade (e.g. índice de cinco ou mais pontos é preditor de morte a três anos, em $85 \%$ dos doentes). ${ }^{7}$ No entanto, a multimorbilidade, a carga de doença e a complexidade do doente não estão ainda bem conceptualizadas. ${ }^{4}$

Além da morbilidade, as pessoas de mais idade experienciam um declínio gradual da força física, da velocidade da marcha, da destreza manual, da memória e das capacidades cognitivas, mesmo na ausência de um processo de doença clinicamente manifesta (fragilidade). ${ }^{6}$ Os conceitos de multimorbilidade e de fragilidade confundem-se no idoso, apesar de não serem permutáveis. ${ }^{8} \mathrm{~A}$ fragilidade do idoso é 'um estado de vulnerabilidade' definido como uma perda progressiva da reserva e capacidade de adaptação, associado a uma deterioração geral da saúde que pode resultar em incapacidade, perda de independência, hospitalização, uso excessivo de recursos de saúde, necessidade de cuidados a longo prazo e morte (Tabela 1). ${ }^{6,8,9}$ Estes doentes são complexos e apresentam frequentemente morbilidade, física e mental, polifarmácia e vulnerabilidade social, requerendo abordagens multidisciplinares em contexto de cuidados de saúde primários (CSP). ${ }^{8}$ Apesar do uso generalizado do termo fragilidade, não há acordo sobre a sua definição ou sobre um instrumento que a identifique de forma simples. ${ }^{9}$ Um estudo considerou fragilidade como perda da independência, medida através da escala de Barthel. Assim, considerou como frágil todo o indivíduo que tivesse uma pontuação de Barthel inferior a 90 (ligeiramente dependente) ${ }^{9}$

Os dados de prevalência de dependência em idosos são limitados e os instrumentos utilizados para a medir são múltiplos, uns medem a dependência nas atividades básicas de vida diária (e.g., comer, vestir, andar), como a escala de Barthel ${ }^{10}$; outros incluem atividades instrumentais (e.g. cozinhar, telefonar, gerir), como a escala de Lawton \& Brody. ${ }^{11}$ Numa análise de uma coorte populacional, no Japão, determinou-se a prevalência e causas de dependência utilizando a escala de Barthel. ${ }^{12}$ Verificou-se uma prevalência de dependência de $20,1 \%$, a qual duplicou a cada cinco anos (e.g. 4,9\% entre os 65 e 69 anos, $9,7 \%$ entre os 70 e 74 anos, até $62,3 \%$ no grupo etário com 85 anos ou mais) e foi superior nas mulheres. ${ }^{12}$

Portugal tem-se adaptado às mudanças sociodemográficas e de necessidades sociais e de saúde, criando e mantendo, desde 2006, a Rede Nacional de Cuidados Continuados Integrados (RNCCl) no âmbito dos Ministérios da Saúde e do Trabalho e da Solidariedade Social. ${ }^{13-15}$ A RNCCl é uma estrutura de articulação intersetorial que cruza cuidados de saúde e apoios sociais e que se caracteriza pela sua descentralização ao nível das regiões de saúde. ${ }^{13-17}$ A prestação de cuidados de saúde e de apoio social é assegurada por diferentes tipologias de cuidados que incluem as equipas domiciliárias [equipas de cuidados continuados integrados (ECCI)], ${ }^{16}$ assumindo-se que os doentes que recebem cuidados no domicílio pelas $\mathrm{ECCl}$ não têm critérios de internamento hospitalar e têm suporte familiar e social que o permita. ${ }^{15,16}$

Decorridos dez anos de atividade da $\mathrm{RNCCl}$, importa conhecer a carga de doença dos doentes integrados em $\mathrm{ECCl}$. Os objetivos definidos neste estudo foram caracterizar, para os doentes com 75 e mais anos, a morbilidade, os determinantes de incapacidade, o grau e a duração de dependência nas atividades básicas de vida diária (escala de Barthel).

\section{MATERIAL E MÉTODOS}

Trata-se de um estudo transversal, descritivo com componente analítica, por entrevista. Por uma questão de exequibilidade definiu-se, como área de abrangência, a região de Lisboa e Vale do Tejo e planeou-se entrevistar os cuidadores dos doentes ao cuidado das ECCI. A população ( $n=324878$ ) corresponde aos idosos com 75 e mais anos da região de Lisboa e Vale do Tejo. ${ }^{18}$ Calculou-se a dimensão da amostra $(n=229)$ tendo em conta a população, a

Tabela 1 - Síntese de conceitos

\section{Multimorbilidade \\ Co-ocorrência, numa pessoa, de duas ou mais doenças ou condições médicas}

\section{Comorbilidade}

Co-ocorrência, numa pessoa, de duas ou mais doenças ou condições médicas, em que uma é definida como problema índice

Fragilidade

Estado de vulnerabilidade definido como uma perda progressiva da reserva e capacidade de adaptação, associado a uma deterioração geral da saúde ligada ao envelhecimento 
prevalência de multimorbilidade $(92 \%)^{19}$ neste grupo etário e um erro alfa de $5 \%$. No sentido de tornar representativa a amostra e não sendo possível aleatorizar os participantes, uma vez que os doentes inseridos em ECCI são uma população dinâmica, realizou-se uma amostragem em duas etapas. A primeira por aleatorização simples das ECCI de forma a garantir a dimensão amostral. Prevendo-se as perdas inerentes à taxa de ocupação das camas em ECCI (esperada uma taxa de ocupação de $60 \%$ ) e ao cumprimento dos critérios de elegibilidade, aleatorizaram-se 25 das 56 $\mathrm{ECCl}$ em funcionamento, o que corresponderia a 829 doentes (número de vagas disponíveis). A segunda etapa de amostragem consistiu na inclusão de todos os idosos que se encontrassem ao cuidado da equipa no período planeado para a colheita de dados que cumprissem os critérios de elegibilidade e que aceitassem participar.

Definiram-se os critérios de elegibilidade:

- De inclusão:

- doente a receber cuidados da $\mathrm{ECCl}$

- com idade igual ou superior a 75 anos;

- que tivesse cuidador não remunerado; e

- que aceitasse participar.

- De exclusão:

- doente institucionalizado à data da entrevista;

- com idade inferior a 75 anos; ou

- que ambos, cuidador e doente, não soubessem ler nem escrever.

Definiu-se como cuidador informal qualquer pessoa que prestasse cuidados gratuitamente por ter uma relação afetiva (e.g. amigos ou vizinhos) ou de parentesco.
A entrevista foi realizada ao cuidador para não limitar a identificação da morbilidade apenas à doença física.

As variáveis estudadas foram as sociodemográficas (idade, género, escolaridade) e as relativas aos objetivos do estudo: determinantes de incapacidade (os que justificaram a incapacidade - dependência de terceiros, reconhecidos pelo cuidador); duração de incapacidade (desde que reconhecida a incapacidade); grau de dependência (escala de dependência nas atividades de vida diária - Barthel); ${ }^{10}$ morbilidade (utilizou-se a escala de Charlson ${ }^{20,21}$ - índice e cada um dos problemas dicotomizados, aos quais se acrescentaram os problemas de saúde mais frequentes na população idosa ${ }^{22}$ e os diagnósticos a reportar pelo cuidador no item - outros).

O questionário foi construído tendo em conta as variáveis que se pretendiam estudar e foi realizado teste de legibilidade, compreensibilidade e aplicabilidade em duas etapas (12 participantes no total, idades entre os 47 e os 79 anos, escolaridade desde 'sem escolaridade' à 'licenciatura').

A colheita de dados ocorreu no domicílio dos doentes, através de entrevista realizada pelo investigador ao cuidador, durante a visita da equipa (ECCI) ou em visita programada com o cuidador. A recolha de dados foi padronizada por aplicação do mesmo instrumento a todos os participantes, pelo investigador. O instrumento previa, à partida, uma pergunta aberta, outros diagnósticos, que foram codificados a posteriori. Na codificação utilizou-se a Classificação Internacional das Doenças para os Cuidados de Saúde Primários (ICPC-2), ${ }^{23}$ pelo contexto onde decorreu o estudo.

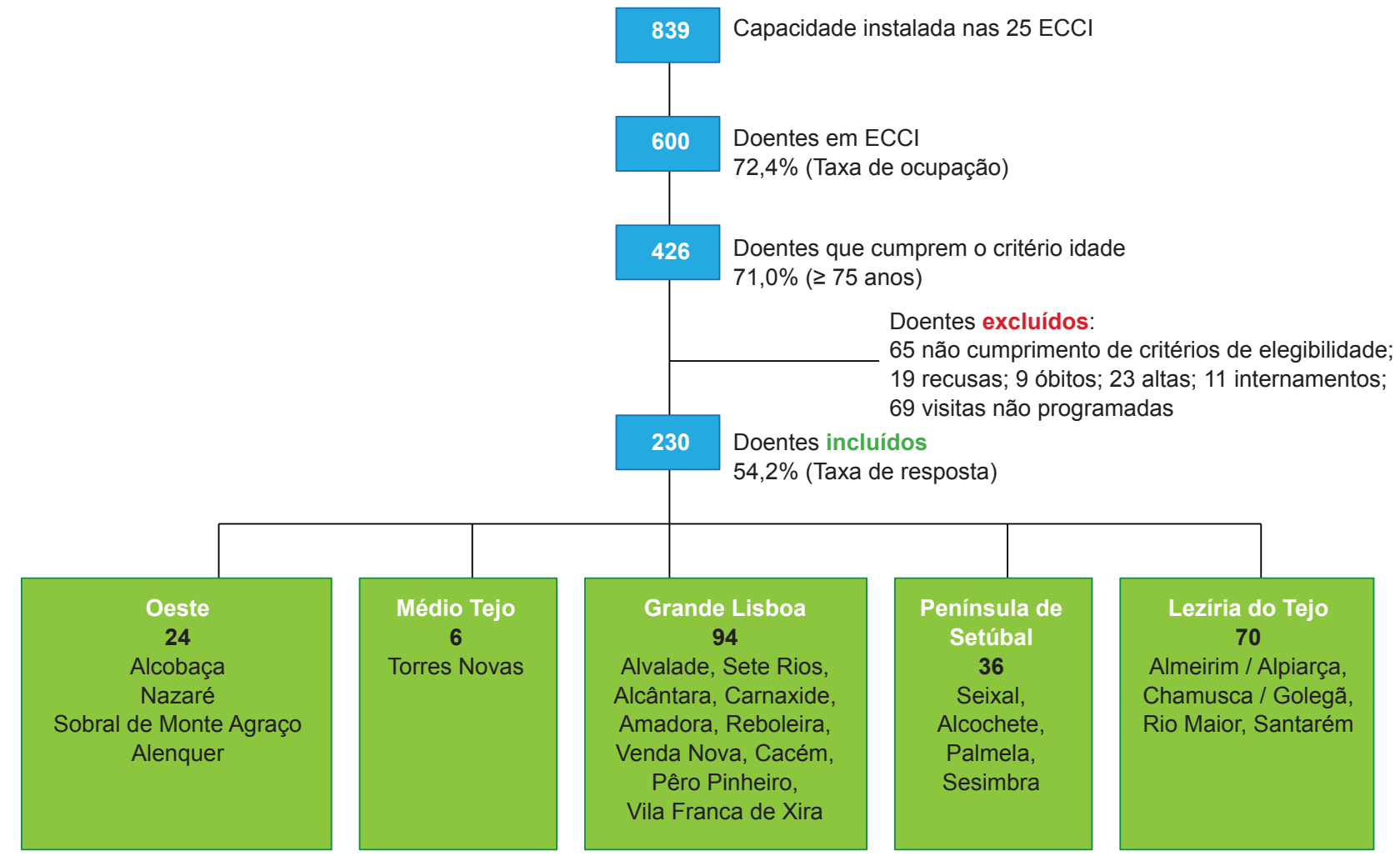

Figura 1 - Fluxograma de cumprimento dos critérios de elegibilidade dos participantes e sua distribuição por regiões 
Tabela 2 - Características sociodemográficas dos idosos

\begin{tabular}{|c|c|}
\hline Idade & Média \\
\hline & 83,59 anos \\
\hline Género & $\begin{array}{l}\text { Feminino (124) } \\
\text { Masculino (106) }\end{array}$ \\
\hline Escolaridade & $\begin{array}{c}\text { Frequência } \\
\text { n (\%) }\end{array}$ \\
\hline Não sabe ler nem escrever & $56(24,3)$ \\
\hline Sabe ler e escrever & $37(16,1)$ \\
\hline $1^{\circ}$ Ciclo & $111(48,3)$ \\
\hline $2^{\circ}$ e $3^{\circ}$ Ciclo & $9(3,9)$ \\
\hline Secundário & $12(5,2)$ \\
\hline Formação superior & $5(2,2)$ \\
\hline
\end{tabular}

Tanto para a questão aberta como para os dados reportados de morbilidade, a confirmação diagnóstica foi feita através da análise da terapêutica em curso e da consulta da informação clínica disponível (e.g. notas de alta hospitalar ou relatórios clínicos).

Durante a entrevista, os dados foram registados num ficheiro word, em seguida exportados para uma matriz excel e, posteriormente, para SPSS v.23.

$\mathrm{Na}$ análise dos dados foram estudadas frequências para variáveis nominais e médias com intervalos de confiança para variáveis numéricas. Para aferir a existência de associação entre variáveis utilizaram-se a correlação de Spearman, o qui-quadrado e a correlação de Pearson.

O estudo obteve parecer favorável da Comissão de Ética para a Saúde da ARSLVT e da Comissão Nacional de Proteção de Dados.

\section{RESULTADOS}

O fluxograma (Fig. 1) sintetiza o cumprimento dos critérios de elegibilidade dos participantes, bem como a distribuição por regiões (NUTS III) dos 230 idosos incluídos na amostra.

As características sociodemográficas dos doentes en- contram-se sumarizadas na Tabela 2. O critério de inclusão idade (75 e mais anos) foi verificado em $71 \%$. Nestes, a média de idades foi de 83,59 anos, verificando-se um predomínio feminino e baixa escolaridade (em $48,3 \%$ escolaridade mínima e em 40,4\% escolaridade abaixo da mínima). Trinta e quatro idosos $(14,8 \%)$ residiam sós.

Os cuidadores foram questionados sobre o problema que identificavam como causa de incapacidade (dependência de terceiros) e desde quando. Assim, identificaram-se os determinantes de incapacidade e a sua data de início (duração aproximada de incapacidade) (Tabela 3). Os mais frequentes foram o acidente vascular cerebral, a fratura do fémur e a demência, sendo que dez determinantes de incapacidade corresponderam a cerca de dois terços $(74,3 \%)$ do total dos motivos de dependência reconhecidos pelos cuidadores. Salienta-se o determinante reportado 'deixou de andar' $(12,2 \%)$ como causa de incapacidade percecionada pelo cuidador, contudo, não se enquadrando numa entidade nosológica.

Em 53,9\% dos participantes verificou-se dependência total nas atividades de vida diária não instrumentais (escala de Barthel) e em 65,2\% dependência grave a total (Tabela 4). A duração média de dependência foi de 42,0 meses [IC 95\%: 33,6 - 50,4], tendo-se verificado associação significativa $(p<0,05)$ entre duração e grau de dependência. O índice médio da escala de Barthel foi de 32,4 [IC 95\%: $27,9-36,9]$, existindo diferença significativa $(p<0,05)$ entre géneros para a dependência total, sendo superior nos homens. Verificou-se uma associação significativa entre a dependência total e idade $(p<0,05)$. Quanto à dependência verificaram-se diferenças significativas entre regiões $(p$ $<0,05)$, sendo a dependência superior na Grande Lisboa e Lezíria (OR $=1,1)$ e inferior na Península de Setúbal e Oeste (OR de 0,9 e 0,7, respetivamente).

Os diagnósticos mais frequentes foram a osteoartrose e a hipertensão arterial, conforme expresso na Tabela 5. Verificaram-se diferenças entre géneros, sendo superior nas mulheres a frequência de osteoartrose, osteoporose

Tabela 3 - Determinantes de incapacidade mais frequentes, por género

\begin{tabular}{|c|c|c|c|c|c|c|c|}
\hline Determinantes de incapacidade ${ }^{a}$ & ICPC2 & $\mathbf{F}$ & $\%$ & $\mathbf{M}$ & $\%$ & Total & $\%$ \\
\hline Acidente vascular cerebral & K90 & 19 & 15,3 & 27 & 25,5 & 46 & 20,0 \\
\hline Fractura do fémur & L75 & 23 & 18,5 & 7 & 6,6 & 30 & 13,0 \\
\hline $\begin{array}{l}\text { Doença do aparelho musculo-esquelética, outra } \\
\text { (deixou de andar) }\end{array}$ & & 18 & 14,5 & 10 & 9,4 & 28 & 12,2 \\
\hline Demência & P70 & 14 & 11,3 & 13 & 12,3 & 27 & 11,7 \\
\hline Úlcera crónica de pele & S97 & 8 & 6,5 & 7 & 6,6 & 15 & 6,5 \\
\hline Neoplasias malignas & & 4 & 3,2 & 6 & 5,7 & 10 & 4,3 \\
\hline Amputação de membro & L98 & 4 & 3,2 & 4 & 3,8 & 8 & 3,5 \\
\hline Lesão craniana & N80 & 5 & 4,0 & 1 & 0,9 & 6 & 2,6 \\
\hline Parkinsonismo & N87 & 2 & 1,6 & 4 & 3,8 & 6 & 2,6 \\
\hline Insuficiência cardíaca & $\mathrm{K} 77$ & 2 & 1,6 & 3 & 2,8 & 5 & 2,2 \\
\hline Outras fraturas & L76 & 2 & 1,6 & 2 & 1,9 & 4 & 1,7 \\
\hline Osteoartrose do joelho & L90 & 2 & 1,6 & 1 & 0,9 & 3 & 1,3 \\
\hline
\end{tabular}

a $\mathrm{O}$ número de determinantes de incapacidade identificados foi 39 no total 
Tabela 4 - Frequência da dependência nas AVD por género e grupo etário

\begin{tabular}{|c|c|c|c|c|c|c|c|c|}
\hline \multirow{2}{*}{$\begin{array}{l}\text { Escala de Barthel } \\
\text { (pontuação) }\end{array}$} & \multicolumn{2}{|c|}{ Género } & \multirow{2}{*}{$\begin{array}{l}\text { Total } \\
\text { (\%) }\end{array}$} & \multicolumn{5}{|c|}{ Grupos etários } \\
\hline & $\mathbf{F}$ & M & & 75 a 79 & 80 a 84 & 85 a 89 & 90 a 94 & 95 a 99 \\
\hline Dependência total (0 a 25) & $\begin{array}{c}75 \\
(60,5)\end{array}$ & $\begin{array}{c}49 \\
(46,2)\end{array}$ & $\begin{array}{c}124 \\
(53,9)\end{array}$ & $\begin{array}{c}30 \\
(44,8)\end{array}$ & $\begin{array}{c}34 \\
(50,7)\end{array}$ & $\begin{array}{c}32 \\
(58,2)\end{array}$ & $\begin{array}{c}18 \\
(64,3)\end{array}$ & $\begin{array}{c}10 \\
(76,9)\end{array}$ \\
\hline Dependência grave (26 a 50) & $\begin{array}{c}9 \\
(7,3)\end{array}$ & $\begin{array}{c}17 \\
(16,0)\end{array}$ & $\begin{array}{c}26 \\
(11,3)\end{array}$ & $\begin{array}{c}8 \\
(11,9)\end{array}$ & $\begin{array}{c}8 \\
(11,9)\end{array}$ & $\begin{array}{c}7 \\
(12,7)\end{array}$ & $\begin{array}{c}2 \\
(7,1)\end{array}$ & $\begin{array}{c}1 \\
(7,7)\end{array}$ \\
\hline Dependência moderada (51 a 75) & $\begin{array}{c}19 \\
(15,3)\end{array}$ & $\begin{array}{c}22 \\
(20,8)\end{array}$ & $\begin{array}{c}41 \\
(17,8)\end{array}$ & $\begin{array}{c}17 \\
(25,4)\end{array}$ & $\begin{array}{c}13 \\
(19,4)\end{array}$ & $\begin{array}{c}6 \\
(10,9)\end{array}$ & $\begin{array}{c}5 \\
(17,9)\end{array}$ & $\begin{array}{c}0 \\
(0,0)\end{array}$ \\
\hline Dependência leve (76 a 99) & $\begin{array}{c}19 \\
(15,3)\end{array}$ & $\begin{array}{c}17 \\
(16,0)\end{array}$ & $\begin{array}{c}36 \\
(15,7)\end{array}$ & $\begin{array}{c}11 \\
(16,4)\end{array}$ & $\begin{array}{c}11 \\
(16,4)\end{array}$ & $\begin{array}{c}9 \\
(16,4)\end{array}$ & $\begin{array}{c}3 \\
(10,7)\end{array}$ & $\begin{array}{c}2 \\
(15,4)\end{array}$ \\
\hline Independência (100) & $\begin{array}{c}2 \\
(1,6)\end{array}$ & $\begin{array}{c}1 \\
(0,9)\end{array}$ & $\begin{array}{c}3 \\
(1,3)\end{array}$ & $\begin{array}{c}1 \\
(1,5)\end{array}$ & $\begin{array}{c}1 \\
(1,5)\end{array}$ & $\begin{array}{c}1 \\
1,8)\end{array}$ & $\begin{array}{c}0 \\
(0,0)\end{array}$ & $\begin{array}{c}0 \\
(0,0)\end{array}$ \\
\hline Total & $\begin{array}{c}124 \\
(100)\end{array}$ & $\begin{array}{c}106 \\
(100)\end{array}$ & $\begin{array}{c}230 \\
(100)\end{array}$ & $\begin{array}{c}67 \\
(100)\end{array}$ & $\begin{array}{c}67 \\
(100)\end{array}$ & $\begin{array}{c}55 \\
(100)\end{array}$ & $\begin{array}{c}28 \\
(100)\end{array}$ & $\begin{array}{c}13 \\
(100)\end{array}$ \\
\hline
\end{tabular}

Tabela 5 - Os 20 diagnósticos mais frequentes segundo ICPC2

\begin{tabular}{|c|c|c|c|}
\hline & Feminino (\%) & Masculino (\%) & Total (\%) \\
\hline Osteoartrose (L89 a L91) & $99(79,8)$ & $76(71,7)$ & $175(76,1)$ \\
\hline Hipertensão arterial (K86 e 87) & $83(66,9)$ & $83(78,3)$ & $166(72,2)$ \\
\hline Demência (P70) & $82(66,1)$ & $62(62,3)$ & $148(64,3)$ \\
\hline Doença vascular cerebral (K91) & $52(41,9)$ & $62(58,5)$ & $114(49,6)$ \\
\hline Perturbação do sono (P06) & $62(50,0)$ & $42(39,6)$ & $106(45,2)$ \\
\hline Doença vascular periférica (K92) & $51(41,1)$ & $49(46,2)$ & $100(43,5)$ \\
\hline Perturbação depressiva (P76) & $42(33,9)$ & $48(45,3)$ & $90(39,1)$ \\
\hline Osteoporose (L95) & $64(51,6)$ & $25(23,6)$ & $89(38,7)$ \\
\hline Insuficiência cardíaca (K77) & $42(33,9)$ & $37(34,9)$ & $79(34,3)$ \\
\hline Distúrbio de ansiedade (P74) & $45(36,3)$ & $32(30,2)$ & $77(33,5)$ \\
\hline Diabetes (T89 e 90) & $39(31,5)$ & $36(34,0)$ & $75(32,6)$ \\
\hline Úlcera crónica da pele (S97) & $36(29,0)$ & $18(17,0)$ & $54(23,5)$ \\
\hline Alteração do metabolismo dos lípidos (T93) & $24(19,4)$ & $22(20,8)$ & $46(20,0)$ \\
\hline Obesidade (T82) & $24(19,4)$ & $21(19,8)$ & $45(19,6)$ \\
\hline Úlcera péptica (D85 e 86) & $17(13,7)$ & $24(22,6)$ & $41(17,8)$ \\
\hline Cancro não metastizado & $14(11,3)$ & $25(23,6)$ & $39(17,0)$ \\
\hline Doença pulmonar obstrutiva crónica (R95) & $7(5,6)$ & $27(25,5)$ & $34(14,8)$ \\
\hline Arritmia cardíaca (K80) & $13(10,5)$ & $16(15,1)$ & $29(12,6)$ \\
\hline Dor generalizada / múltipla (A01) & $12(9,7)$ & $9(8,5)$ & $21(9,1)$ \\
\hline Fibrilhação auricular (K78) & $10(8,1)$ & $10(9,4)$ & $20(8,7)$ \\
\hline
\end{tabular}

e úlcera crónica da pele. Nos homens, a hipertensão, a doença vascular cerebral, o cancro não metastizado e a doença pulmonar obstrutiva crónica (DPOC) foram mais frequentes do que nas mulheres. A diferença entre géneros foi significativa $(p<0,05)$ para doença vascular cerebral, osteoporose, DPOC, úlcera crónica da pele e cancro não metastizado. Os aparelhos e sistemas mais afetados foram o circulatório; psicológico; músculoesquelético; endócrino, metabólico e nutricional e neurológico [K;P;L;T;N]. Realça-se o neurológico por nenhum dos diagnósticos surgir nos 20 primeiros, sendo que os mais frequentes foram: hemiplegia, epilepsia e parkinsonismo.

Todos os doentes tinham multimorbilidade (Fig. 2) com número médio de problemas por pessoa de 9,5 [IC 95\%: 9,1 - 9,9], nove nas mulheres e 10 nos homens, confirman- do-se existir correlação com o género $(p<0,05)$. O índice médio de comorbilidade de Charlson foi de 8,48 [IC 95\%: 8,14 - 8,83], no género feminino de 7,96 [IC 95\%: 7,5 - 8,42] e no masculino de 9,09 [IC 95\%: 8,59 - 9,6], sendo esta diferença significativa como se infere pelos intervalos de confiança por género.

Relativamente à idade (Fig. 2), não se encontrou associação significativa com o número de problemas, verificando-se um aparente decréscimo no número de diagnósticos nos grupos etários dos mais idosos [9,46 (75 a 79 anos); 9,75 (80 a 84 anos); 9,96 (85 a 89 anos); 8,71 (90 a 94 anos); 7,92 (95 a 99 anos)].

\section{DISCUSSÃO}

A população ao cuidado das $\mathrm{ECCl}$ da $\mathrm{RNCCl}$ é muito 

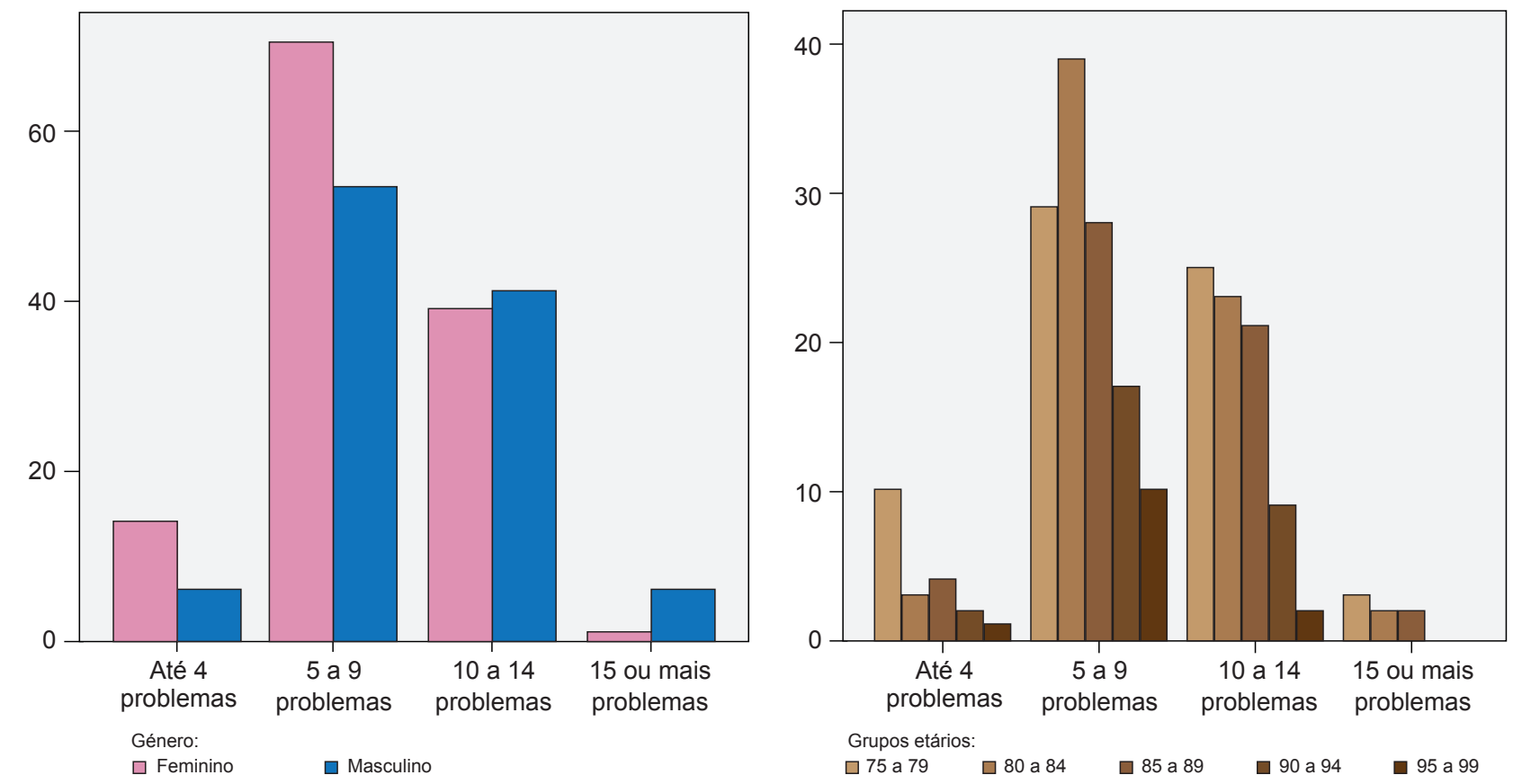

Figura 2 - Multimorbilidade por género e grupo etário

O número médio de problemas por pessoa foi de 9,5 [IC 95\%: 9,1 - 9,9], superior nos homens, sendo esta correlação para o género significativa ( $p<0,05)$. A correlação de multimorbilidade com a idade não foi significativa.

O valor médio do índice de Charlson foi de 8,48 [IC 95\%: 8,14 - 8,83], de 7,96 [IC 95\%: 7,50 - 8,42] no género feminino e superior no género masculino 9,09 [IC 95\%: 8,59 - 9,60].

idosa, o que se infere dos $71 \%$ dos participantes que cumpriam o critério de inclusão idade (75 e mais anos) (Fig. 1), com predomínio feminino, à semelhança do ocorrido na Europa. ${ }^{24,25}$ A média de idades dos participantes foi de 83,59 anos, $88,7 \%$ tinham baixa escolaridade $(40,4 \%$ escolaridade abaixo da mínima e 48,3\% escolaridade mínima) e cerca de $15 \%$ residiam sós. A educação é considerada um indicador económico, ${ }^{26}$ no entanto, tratando-se de uma amostra predominantemente feminina e idosa não se pode, por razões de natureza sociocultural, inferir com segurança tratar-se de uma população de baixo estrato social. Na nossa amostra, 53,9\% dos participantes tinham dependência total e $65,2 \%$ dependência grave a total (Tabela 3). Estes resultados parecem confirmar os dados Europeus de que o aumento da esperança de vida não conduziu necessariamente a um aumento de anos de vida saudável, mas, pelo contrário, a uma proporção de vida remanescente vivida com limitação funcional ou incapacidade. ${ }^{25} \mathrm{~A}$ proporção de casos com dependência é superior no nosso estudo por comparação ao estudo de base populacional japonês $(20,1 \%),{ }^{12}$ o que é justificável pelos critérios de inclusão na amostra: doentes com idade igual ou superior a 75 anos, com cuidador e a receber cuidados da ECCI. Verificaram-se associações significativas $(p<0,05)$ entre género, idade e gravidade da dependência, o que confere validade interna aos resultados.

O problema 'deixou de andar', identificado pelo cuidador como determinante de incapacidade que justificou a incapacidade (dependência de terceiros), foi o que mais nos surpreendeu. Contudo, revendo o fenótipo da fragilidade no idoso (perda de peso, cansaço, fraqueza, baixa atividade fí- sica e dificuldade de mobilidade $)^{6,27,28}$ e os sinais e sintomas não-cognitivos de demência (alteração da marcha e do equilíbrio), ${ }^{29,30}$ os resultados parecem refletir a coexistência do declínio da função motora e o envelhecimento e/ou a demência (declínio cognitivo). ${ }^{31}$ A marcha era considerada como uma tarefa motora automática; todavia, a evidência emergente sugere que é uma atividade complexa que exige capacidades físicas (e.g. músculoesquelética, vascular, endocrinológica), amnésicas e motoras, como equilíbrio e coordenação. ${ }^{29,31,32}$ A complexidade das alterações motoras nos idosos pode ser objetivada: tempo de passo, velocidade de marcha ou dupla tarefa (e.g. falar e andar). ${ }^{33}$ Alterações relacionadas com a idade, como diminuição da força muscular, sarcopenia e alterações sensoriais (e.g. proprioceção, visão e audição) prejudicam o controlo postural. ${ }^{32} \mathrm{~A}$ ocorrência de alterações da marcha pode ajudar a identificar o risco de declínio da mobilidade, quedas e progressão para a demência. $O$ inverso também é verdadeiro: pessoas com défices cognitivos que, com treino específico de atenção e função executiva, podem retardar as alterações da mobilidade e diminuir o risco de queda. ${ }^{31,32}$ Se se interpretar o evento 'deixou de andar' como uma manifestação não-cognitiva de demência poder-se-á assumir que os sinais e sintomas demenciais são a causa de incapacidade, reconhecida pelos cuidadores, mais frequente $(24,9 \%)$.

As fraturas estão a tornar-se um dos problemas de saúde mais prevalentes associados ao envelhecimento da população. ${ }^{34}$ As fraturas têm sido consideradas um problema de saúde pública pelo impacto prejudicial na saúde física e mental dos doentes e por contribuírem para a carga de morbilidade, mortalidade e necessidade de cuidados de 
saúde. ${ }^{34}$ A osteoporose é um fator de risco conhecido que, associado à síndroma de fragilidade do idoso, pode agravar essa vulnerabilidade. Numa revisão sistemática, apesar da heterogeneidade dos estudos, verificou-se que indivíduos classificados como frágeis tinham risco acrescido de fratura em comparação com os não-frágeis. ${ }^{34}$ Contudo, os mecanismos exatos subjacentes à associação entre fragilidade e um maior risco de fratura não são claros, considerando-se essa associação complexa e multifatorial. ${ }^{34}$ Às alterações da marcha acresce o risco de queda cujas consequências, fratura do fémur $(13 \%)$ e traumatismo craniano $(2,6 \%)$, identificados no estudo como determinantes de incapacidade, podem ser o seu reflexo.

Quanto às causas de incapacidade, os resultados [demência $(23,9 \%)$, acidente vascular cerebral $(20 \%)$ e fraturas $(13 \%)]$ são comparáveis aos do estudo japonês que refere como causas de incapacidade funcional mais frequentes: a demência $(23,5 \%)$, o acidente vascular cerebral $(24,7 \%)$ e a doença ortopédica $(12,9 \%){ }^{12}$

Relativamente aos diagnósticos mais frequentes (Tabela 5) verificou-se semelhança com os identificados noutros estudos realizados em diferentes contextos (comunidade ou internamento), ${ }^{22,35,36}$ independentemente da idade. ${ }^{37,38}$ Dentro de cada aparelho ou sistema, os problemas mais frequentes na amostra foram semelhantes aos encontrados num outro estudo sobre carga de doença realizado no Reino Unido em idosos na comunidade. ${ }^{39}$ Os dados do EUROSTAT confirmam como problemas mais frequentes do ambulatório a hipertensão, a osteoartrose e a diabetes. ${ }^{40}$ Por órgãos e sistemas também houve coincidência com os dados Europeus: hipertensão, doença vascular cerebral, demência, osteoartrose, osteoporose, diabetes, epilepsia e parkinsonismo. ${ }^{40}$

A úlcera crónica de pele encontra-se entre as cinco primeiras causas reportadas como causa de dependência e encontra-se entre os 12 diagnósticos mais frequentes $(23,5 \%)$ na amostra, semelhante à encontrada num estudo de prevalência realizado numa unidade de longa duração. ${ }^{35,41}$ A DPOC também se encontra nos 20 problemas mais frequentes, verificando-se uma associação significativa ao género masculino, revelando coerência com os dados da literatura. ${ }^{42}$

Uma questão suscitada pela análise da Tabela 5 foi a frequência de demência $(64,3 \%)$ e de depressão $(39,1 \%)$ na amostra. Atendendo a que a colheita de dados foi complementada com a terapêutica considerou-se poder existir um eventual sobrerregisto pela manutenção da terapêutica antidepressiva. Confrontando com os dados da literatura, confirma-se que a depressão na demência é frequente em todos os seus estádios (20 a 30\%), ${ }^{43}$ sendo mais elevada na demência vascular $(42,9 \%) .{ }^{43}$

Neste estudo encontrou-se uma elevada carga de doença que se refletiu numa média de problemas por pessoa de 9,5 [IC 95\%: 9,1 - 9,9] e num índice de Charlson de 8,48 [IC 95\%: 8,14 - 8,83]. Como se pode observar na Fig. 2 verificou-se associação entre carga de doença (número de problemas) e género masculino, ao contrário do eviden- ciado na literatura..$^{44,45}$

Quando os estudos foram realizados na população verificou-se diferença entre grupos etários, sendo a multimorbilidade superior nos idosos. ${ }^{45}$ Como o estudo foi realizado com idosos não se encontrou diferença entre grupos etários, parecendo mesmo ser menos frequente no grupo dos mais idosos (90 e mais anos). Estes dados reforçam a necessidade de colocar em questão a idade como proxi de carga de doença na população. ${ }^{6}$

Neste estudo, as pessoas que vivem mais parecem ter menor multimorbilidade (número de diagnósticos); contudo, são mais dependentes. Um estudo sueco, numa população com 60 e mais anos, teve como objetivos avaliar o desempenho físico associado à idade e explorar as diferenças atribuíveis à multimorbilidade. A multimorbilidade esteve associada a pior desempenho físico, particularmente nos homens mais jovens e explicou apenas parte das diferenças atribuíveis à idade. ${ }^{46} \mathrm{~A}$ aparente menor multimorbilidade nos mais idosos, a par de maior dependência associada à idade, merece reflexão e outros estudos que melhor clarifiquem a fronteira entre multimorbilidade e fragilidade e/ou dependência no grande idoso.

Os resultados obtidos revelam coerência interna do estudo, de que é exemplo a associação entre gravidade e duração de dependência, idade e gravidade da dependência e também coerência externa, objetivada nas semelhanças, atrás descritas, com os dados da literatura. Como limitações há a referir:

- a dimensão amostral e a sua representatividade, particularmente, dos idosos acima dos 90 anos;

- o apuramento dos diagnósticos (reportados, ainda que complementados com informação terapêutica e informação clínica);

- a utilização da escala de AVD de Barthel, por não incluir atividades instrumentais.

Importa também sublinhar alguns aspectos que distinguem este estudo:

- os resultados corresponderem a dados do ambulatório, provenientes das equipas de cuidados domiciliários integrados da RNCCl;

- a população incluída é a do grupo dos grandes idosos (75 e mais anos), excluída do Inquérito Nacional de Saúde com exame objectivo ${ }^{37}$ e que na literatura se encontra incluída no grupo dos 65 anos e mais anos, ou não desagregada após os 85 anos;

- é caraterizada a carga de doença, em contexto extra-hospitalar, através do índice de Charlson e do conceito de multimorbilidade.

Verificou-se que os idosos com 90 e mais anos tinham menor número de diagnósticos o que merecerá estudos futuros. Carga de doença e multimorbilidade constituem áreas a necessitar de investigação. ${ }^{47} \mathrm{O}$ conhecimento sobre a carga de doença e dependência na população idosa ao cuidado das ECCI poderá ser útil para a reflexão sobre o modelo organizativo de cuidados e consequente alocação de recursos. 
Seria, pois, interessante um estudo de base populacional que avaliasse a morbilidade e dependência dos idosos na população portuguesa. Esta população muito idosa, com multimorbilidade e elevada dependência, sem critérios de internamento hospitalar, parece trazer novos desafios clínicos (e.g. aplicabilidade individual da evidência disponível) e organizacionais (e.g. prestação de cuidados no domicílio).

\section{CONCLUSÃO}

A população em estudo ao cuidado das ECCl é constituída, maioritariamente, por grandes idosos (83,59 anos), com escolaridade baixa ou ausente $(88,7 \%)$, com elevada carga de doença (número médio de problemas por pessoa - 9,5 e 8,48 de índice de comorbilidade de Charlson) e dependente $(65,2 \%$ dependência grave a total). Os determinantes de incapacidade mais frequentes foram: acidente vascular cerebral, fratura do fémur e 'demência'. As patologias mais frequentes foram as mais prevalentes na população (osteoartrose e hipertensão) e as associadas à idade (demência). Na população idosa, a multimorbilidade, afetando vários aparelhos/sistemas, a par do elevado grau de dependência, traz novos desafios clínicos (e.g. aplicabilidade da evidência) e organizacionais (e.g. prestação de cuidados no domicílio).

\section{AGRADECIMENTOS}

Agradeço a Regina Sequeira Carlos, Coordenadora da

\section{REFERÊNCIAS}

1. Kaplan W, Wirtz V, Mantel-Teeuwisse A, Stolk P, Duthey B, Laing R. Priority medicines for Europe and the World 2013 update. Geneva: WHO Library; 2013.

2. Instituto Nacional de Estatística. 25 de Abril - 40 anos de estatísticas. Lisboa: INE; 2014.

3. Instituto Nacional de Estatística. Estatísticas demográficas, 2013. Lisboa: INE; 2014.

4. Valderas JM, Starfi B, Sibbald B. Understanding health and health services. Ann Fam Med. 2009;7:357-63.

5. Broeiro P. Multimorbilidade e comorbilidade: duas perspectivas da mesma realidade. Rev Port Med Geral Fam. 2015;31:158-60.

6. Karlamangla A, Tinetti M, Guralnik J, Studenski S, Wetle T, Reuben D. Comorbidity in older adults: nosology of impairment, diseases, and conditions. J Gerontol Med Sci. 2007;62:296-300.

7. De Groot V, Beckerman H, Lankhorst GJ, Bouter LM. How to measure comorbidity: a critical review of available methods. J Clin Epidemiol. 2003;56:221-9.

8. Romero-Ortuno R, Soraghan C. A frailty instrument for primary care for those aged 75 years or more: findings from the Survey of Health, Ageing and Retirement in Europe, a longitudinal population-based cohort study (SHARE-FI75+). BMJ Open. 2014;4:e006645.

9. Diez-Ruiz A, Bueno-Errandonea A, Nuñez-Barrio J, Sanchez-Martín I, Vrotsou K, Vergara I. Factors associated with frailty in primary care: a prospective cohort study. BMC Geriatr. 2016;16:91.

10. Araújo F, Oliveira A, Pinto $C$, Ribeiro J. Validação do índice de Barthel numa amostra de idosos não institucionalizados. Rev Port Saúde Pública. 2007;25:59-66.

11. Araújo F, Ribeiro JP, Oliveira A, Pinto C, Martins T. Validação da escala de Lawton e Brody numa amostra de idosos não institucionalizados. In: Leal I, Pais-Ribeiro JL, Silva I, Marques S, editores. Actas do $7^{\circ}$ Congresso Nacional de Psicologia da Saúde. Lisboa: ISPA; 2008. p. 217-20.

12. Yoshida D, Ninomiya T, Doi Y, Hata J, Fukuhara M, Ikeda F, et al. Prevalence and causes of functional disability in an elderly general
Equipa Coordenadora Regional de Lisboa e Vale do Tejo da Rede Nacional de Cuidados Continuados Integrados, pelo incentivo; a todos os coordenadores das equipas coordenadoras locais e a todos os profissionais que integram as equipas de cuidados continuados integrados visitadas na região de Lisboa e Vale do Tejo, pela disponibilidade e recetividade.

Agradeço ao meu orientador, Pedro Aguiar, pela sua supervisão e crítica atenta.

\section{PROTECÇÃO DE PESSOAS E ANIMAIS}

Os autores declaram que os procedimentos seguidos estavam de acordo com os regulamentos estabelecidos pelos responsáveis da Comissão de Investigação Clínica e Ética e de acordo com a Declaração de Helsínquia da Associação Médica Mundial.

\section{CONFIDENCIALIDADE DOS DADOS}

Os autores declaram ter seguido os protocolos do seu centro de trabalho acerca da publicação de dados.

\section{CONFLITOS DE INTERESSE}

Os autores declaram não terem qualquer conflito de interesse relativamente ao presente artigo.

\section{FONTES DE FINANCIAMENTO}

Os autores declaram não ter recebido subsídios ou bolsas para a elaboração do artigo.

population of Japanese: the Hisayama Study. J Epidemiol. 2012;22:222 9.

13. Portugal. Decreto-Lei n 101/2006, de 6 de junho. Diário da República. $1^{\text {a }}$ Série-A (109).

14. Missão para os Cuidados de Saúde Primários. Cuidados continuados integrados nos cuidados de saúde primários: carteira de serviços. Lisboa: Ministério da Saúde; 2007.

15. Lopes M, Mendes F, Escoval A, Agostinho M, Vieira C, Vieira I, et al. Plano nacional de saúde 2011-2016: cuidados continuados integrados - analisando o presente, perspectivando o futuro [Internet]. Évora: Centro de Investigação em Ciências e Tecnologias da Saúde da Universidade de Évora; 2010. [consultado 2015 fev 15]. Disponível em: http://1nj5ms2lli5hdggbe3mm7ms5.wpengine.netdna-cdn.com/ files/2010/08/CSC1.pdf.

16. Unidade de Missão para os Cuidados Continuados Integrados. Manual do prestador: recomendações para a melhoria contínua [Internet] Lisboa: UMCCI; 2011. [consultado 2015 jan 22]. Disponível em: http://www.seg-social.pt/documents/10152/3735095/Man_Prestador_ UMCCI-RNCCI/fe2e98b2-35ba-4a7a-9eb9-d3bba713e7b5.

17. Missão para os Cuidados de Saúde Primários. A equipa de cuidados continuados integrados: orientações para a sua constituição nos centros de saúde [Internet]. Lisboa: MCSP; 2007. [consultado 2015 fev 15]. Disponível em: http://www.acss.min-saude.pt/Portals/0/ Orienta\%C3\%A7\%C3\%B5es\%20para\%20a\%20consti.pdf.

18. Tavares A, Coelho MA, Rascôa CL. Perfil de saúde 2015 e seus determinantes da Região de Lisboa e Vale do Tejo 2015 (Vol. 1). Lisboa: ARSLVT; 2015.

19. Prazeres F, Santiago L. Prevalence of multimorbidity in the adult population attending primary care in Portugal: a cross-sectional study. BMJ Open. 2015;5:e009287.

20. Charlson M, Szatrowski TP, Peterson J, Gold J. Validation of a combined comorbidity index. J Clin Epidemiol. 1994;47:1245-51.

21. Quan H, Li B, Couris CM, Fushimi K, Graham P, Hider P, et al. Updating and validating the Charlson comorbidity index and score for risk 
adjustment in hospital discharge abstracts using data from 6 countries. Am J Epidemiol. 2011;173:676-82.

22. Koller D, Schön G, Schäfer I, Glaeske G, van den Bussche H, Hansen $\mathrm{H}$. Multimorbidity and long-term care dependency: a five-year follow-up. BMC Geriatr. 2014;14:70.

23. World Organization of National Colleges Academies. Classificação internacional de cuidados de saúde primários [Internet]. $2^{\mathrm{a}}$ ed. Lisboa: Administração Central do Sistema de Saúde; 2011. [consultado 2014 dez 10]. Disponível em: http://www.acss.min-saude.pt/Portals/0/ apmcg_ICPC\%20v\%201.7.pdf.

24. Gabinete de Estatísticas da União Europeia. International Day of Older Persons - Nearly 27 million people aged 80 or over in the European Union: almost 10 years' life expectancy at the age of 80 [Internet]. Luxembourg: Publications Office of the European Union; 2016. [consultado 2017 fev 11]. Disponível em: http://ec.europa.eu/eurostat/ documents/2995521/7672228/3-29092016-AP-EN.pdf/4b90f6bb-43c145ed-985b-dfbe9564157a.

25. Gabinete de Estatísticas da União Europeia. Sustainable development in the European Union: 2015 monitoring report of the UE sustainable development strategy [Internet]. Luxembourg: Publications Office of the European Union; 2015. ISBN 9789279493911. [consultado 2017 fev 11]. Disponível em: http://ec.europa.eu/eurostat/ documents/3217494/6975281/KS-GT-15-001-EN-N.pdf.

26. Organização de Cooperação e de Desenvolvimento Económicos. What are the social benefits of education? Educ Indic Focus [Internet]. 2013;(10). [consultado 2016 jul 26]. Disponível em: http:// www.oecd-ilibrary.org/education/what-are-the-social-benefits-ofeducation_5k4ddxnl39vk-en.

27. Strandberg TE, Pitkälä KH, Tilvis RS. Frailty in older people. Eur Geriatr Med. 2011;2:344-55.

28. Chang SF, Lin PL. Frail phenotype and mortality prediction: a systematic review and meta-analysis of prospective cohort studies. Int J Nurs Stud. 2015;52:1362-74

29. Raudino F. Non-cognitive symptoms and related conditions in the Alzheimer's disease: a literature review. Neurol Sci. 2013;34:1275-82.

30. Buchman AS, Bennett DA. Loss of motor function in preclinical Alzheimer's disease. Exp Rev Neurother. 2011;11:665-76.

31. Montero-Odasso M, Verghese J, Beauchet O, Hausdorff J. Gait and cognition: a complementary approach to understanding brain function and the risk of falling. J Am Geriatr Soc. 2012;60:2127-36.

32. Bridenbaugh SA, Kressig RW. Motor cognitive dual tasking. Z Gerontol Geriatr. 2015;48:15-21.

33. MacAulay RK, Brouillette RM, Foil HC, Bruce-Keller AJ, Keller JN. A longitudinal study on dual-tasking effects on gait: cognitive change predicts gait variance in the elderly. PLoS One. 2014;9:e99436.

34. Kojima G. Frailty as a predictor of future falls among community-dwelling older people: a systematic review and meta-analysis. J Am Med Dir Assoc. 2015;16:1027-33.
35. Clerencia-Sierra M, Calderón-Larrañaga A, Martínez-Velilla N, VergaraMitxeltorena I, Aldaz-Herce P, Poblador-Plou B, et al. Multimorbidity patterns in hospitalized older patients: associations among chronic diseases and geriatric syndromes. PLoS One. 2015;10:e0132909.

36. Hansen H, Schäfer I, Schön G, Riedel-Heller S, Gensichen J, Weyerer $S$, et al. Agreement between self-reported and general practitionerreported chronic conditions among multimorbid patients in primary care: results of the MultiCare Cohort Study. BMC Fam Pract. 2014;15:39.

37. Instituto Nacional de Saúde Dr. Ricardo Jorge. Primeiro inquérito nacional de saúde com exame físico (INSEF): sumário e considerações finais [Internet]. Lisboa: INSA; 2016. [consultado 2015 mar 09]. Disponivel em: http://www.insef.pt/Portugues/Documents/INSEF SumarioRelatorio.pdf.

38. Instituto Nacional de Estatística, Instituto Nacional de Saúde Dr. Ricardo Jorge. Inquérito nacional de saúde 2005/2006 [Internet]. Lisboa: INE, INSA; 2009. ISBN 9789726738458. [consultado 2015 mar 09]. Disponível em: http://www1.arslvt.min-saude.pt/SiteCollectionDocuments/ Planos\%20e\%20Relat\%C3\%B3rios/INS_05_06.pdf.

39. Parker L, Moran GM, Roberts LM, Calvert M, McCahon D. The burden of common chronic disease on health-related quality of life in an elderly community-dwelling population in the UK. Fam Pract. 2014;31:557-63.

40. Gabinete de Estatísticas da União Europeia. Morbidity statistics in the EU: report on pilot studies [Internet]. Luxembourg: Publications Office of the European Union; 2014. ISBN 9789279368868. [consultado 2017 jan 31]. Disponível em: http://ec.europa.eu/eurostat/ documents/3888793/5858521/KS-TC-14-003-EN-N.pdf/bd959e6e10ed-4078-915e-308941c02811

41. Pini LR. Prevalência, risco e prevenção de úlcera de pressão em unidades de cuidados de longa duração [Dissertation]. Porto: FMUP 2012.

42. Dal Negro RW, Bonadiman L, Turco P. Prevalence of different comorbidities in COPD patients by gender and GOLD stage. Multidiscip Respir Med. 2015;10:24.

43. Enache D, Winblad B, Aarsland D. Depression in dementia. Curr Opin Psychiatry. 2011;24:461-72.

44. Abad-Díez JM, Calderón-Larrañaga A, Poncel-Falcó A, PobladorPlou B, Calderón-Meza JM, Sicras-Mainar A, et al. Age and gender differences in the prevalence and patterns of multimorbidity in the older population. BMC Geriatr. 2014;14:75.

45. Rizza A, Kaplan V, Senn O, Rosemann T, Bhend H, Tandjung R. Ageand gender-related prevalence of multimorbidity in primary care: the Swiss FIRE project. BMC Fam Pract. 2012;13:113.

46. Welmer AK, Kareholt I, Angleman S, Rydwik E, Fratiglioni L. Can chronic multimorbidity explain the age-related differences in strength, speed and balance in older adults? Aging Clin Exp Res. 2012;24:480-9.

47. Harbers $M$, Achterberg P. Europeans of retirement age: chronic diseases and economic activity. Vol. 1, EAHC/2010/Health/01 (Lot 1). Brussels: European Commission; 2012 